\title{
Differenz
}

Revista internacional de estudios heideggerianos y sus derivas contemporáneas

AÑO 3 NÚMERO 2: JULIO DE 2016. E-ISSN: 2386-4877 - DOI: 10.12795/Differenz.2016.i02.01

[pp. 11-27]

Recibido: 12/05/2016

Aceptado: 14/06/2016

\section{Hacia una reivindicación de la espacialidad como factor determinante y fundacional de la estructura del Dasein.}

\section{Towards a claim of spatiality as a determining and foundational factor of the structure of Dasein}

\author{
Leticia Basso Monteverde \\ Universidad de Buenos Aires
}

\section{Resumen:}

En este trabajo nos introduciremos en algunos escritos y cursos de Martin Heidegger del período de Marburgo (1923-1928) para examinar la noción de espacialidad (Räumlichkeit) en su sentido existencial. A propósito, pretendemos dilucidar la función metodológica que cumple esta noción en la estructura del Dasein. Con esta intención, analizaremos ciertos datos que nos llevan a advertir que en la "analítica existencial" hay una fuerte tendencia a exponer la cuestión en clave topológica. Pues bien, la caracterización que el filósofo ofrece del hombre, el mundo y el sentido de lo dado invitan a descubrir al ser en una modalidad y contenido puntuales de manifestación que refieren al plano sensible y refuerzan su carácter situacional.

Palabras Clave: Dasein; analítica existencial; espacialidad; mundo; sentido objetivo. 


\section{Abstract:}

In this paper we introduce in some Martin Heidegger's writings and courses from the Marburger Period (1923-1928) to examine the notion of spatiality (Räumlichkeit) in its existential sense. By the way, we aim to elucidate the methodological role of this notion in the structure of Dasein. With this intention, we will analyze some data that lead us to realize that in the "existential analysis" there is a strong tendency to expose the issue in a topological key. Well, the characterization that the philosopher offers of man, the world and the sense of the given invite us to discover being in a specific form and content of manifestation that refer to sensitive plane and reinforce their situational character.

Keywords: Dasein; existential analysis; spatiality; world; objective sense.

\section{Introducción: el rol de la espacialidad en la estructura del Dasein.}

En este trabajo nos introduciremos en los textos y los cursos de Martin Heidegger del período de Marburgo (1923-1928) que comprenden el llamado "círculo de Sein und Zeit" -principalmente en el curso que dicta en 1925, Prolegomena zur Geschichte des Zeitbegriffs- para examinar la noción de espacialidad (Räumlichkeit) en su sentido existencial ${ }^{1}$. A propósito, pretendemos dilucidar la función metodológica que cumple esta noción en la estructura del Dasein. Con esta intención, procederemos a reunir y articular algunos datos que nos llevan a advertir que, de hecho, en la concepción heideggeriana de una "analítica existencial" hay una fuerte tendencia a exponer la cuestión en clave topológica.

Lamentablemente esta tendencia se ve opacada por el ímpetu del filósofo de fundamentar la estructura del Dasein y su comprensión del ser en la temporalidad (Zeitlichkeit). Más allá de esta conclusión apresurada, no podemos esquivar el hecho de que la manera elegida por Heidegger para presentar cómo el Dasein se define a través de su relación con lo dado implica, precisamente, ahondar en una serie de comportamientos que se enraízan en la materialidad de su entorno (Umwelt). Este será el punto clave desde el cual defenderemos la función determinante de la espacialidad. Pues bien, hay una serie de conceptos e imágenes topológicas que aportan una visión particular

1 Heidegger aclara en Sein und Zeit y los cursos en torno a esta gran obra que le interesa investigar un sentido de espacialidad diferente al que la tradición ha dejado asentado por medio del uso de nociones de las ciencias naturales, específicamente de la física. Cuando plantea el sentido de una espacialidad del Dasein intenta distinguirla de esta versión que considera derivada u objetivada $y$, por esto, expone - de una manera un tanto confusa y, por momentos, ambigua- otro sentido ontológico del que la primera deriva y que presenta en términos existenciales. Autores como Hubert DREYFUS (1996) y Jeff MALPAS (2006) Ilaman a este sentido "existencial". 
de la experiencia como realización concreta de la vida en su expresión primaria; previa al posicionamiento teórico que altera (o desmundaniza, en palabras de Heidegger) el encuentro directo con las cosas. Por consiguiente, un acercamiento a esta cuestión es relevante ya que generalmente la espacialidad pasa desapercibida, dada la preeminencia que tiene la recepción e interpretación de la temporalidad -incluso según el proyecto del propio Heidegger².

Si realizamos un seguimiento de su elaboración en esta primera etapa de su obra observaremos que la espacialidad opera de forma encubierta en la constitución del Dasein, adquiriendo un sentido elemental que se acentúa y explicita a medida que prosigue su camino fenomenológico. En efecto, la caracterización que el filósofo ofrece del hombre, el mundo y el sentido de lo dado invitan a descubrir al ser en una modalidad y contenido puntuales de manifestación que refieren al plano sensible y refuerzan su carácter situacional. Esto se debe a que el tono existencial de su concepción e, incluso, el tratamiento metodológico que logra a partir de la postura deíctica de la "indicación formal" muestran cómo se da la estructura bajo una faceta patente de la misma, más que latente. Esta es la consecuencia de la transformación fenomenológica que invierte el recorrido husserliano al fundar lo trascendental en lo fáctico. Habidas cuentas, por más que Heidegger tenga la pretensión de exponer cómo acontece o se temporaliza el ser, para acceder a ello es imprescindible vislumbrar el proceso en una dación concreta -que en Sein und Zeit se emplaza en el Dasein.

A propósito, vale rescatar que la "analítica existencial" puede entenderse como otra manera de abordar la subjetividad. Heidegger va más allá de la perspectiva modernognoseológica que la hipostasia a través de la distinción sujeto-objeto, alma-cuerpo, hombre-mundo, generando un predominio de lo puro y racional. Por el contrario, el filósofo presenta al Dasein como el ente que "es-en-el-mundo" (In-der-Welt-Sein) y que se define por su comportamiento en un escenario específico y frente a lo que se le

2 Jeff Malpas explica al respecto que: "La priorización de la temporalidad en Ser y Tiempo significa que la espacialidad no puede tener un rol fundamental en la estructura del ser-ahí, y en la medida en que sólo la temporalidad es tomada como determinante, esto mismo podría aplicarse a la noción de lugar. De hecho, el proyecto de Heidegger en Ser y Tiempo parece querer mostrar el camino por el cual el lugar y el espacio son ambos, de cierta manera, dependientes del tiempo. Sin embargo la espacialidad constantemente se entromete en la consideración de Heidegger" (Heidegger's Topology. Being, Place, World, Cambridge: The MIT Press, 2006, p. 66). En este sentido, hay una tensión constante en el pensamiento del filósofo acerca del aporte de la espacialidad a la estructura del Dasein, pues algunas veces la considera irrelevante y, otras tantas, absolutamente necesaria. Sabemos que el tema de la espacialidad, como dice Malpas (op.cit., p. 82), no está en el corazón del proyecto heideggeriano pero no podemos evitar afirmar que el tratamiento problemático que se abre con la definición del tipo de espacialidad aludida marca la manera como el filósofo comprende la estructura del Dasein. 
presenta. Sobre este asunto conviene subrayar que el Dasein no es una entidad aislada y, de ante mano, consumada a partir de la cual se instala y consolida el sentido del mundo. Heidegger considera que el Dasein se hace mediante la actividad cotidiana de la vida; ni es independiente de la experiencia ni la condiciona de manera exclusiva. Al respecto dice en el curso de 1925:

No se trata de concebir al Dasein determinando cuáles hayan de ser sus metas y fines, ni en cuanto 'homo' ni a la luz de idea alguna de 'humanidad'; lo que hay que hacer, más bien, es poner al descubierto su manera de ser en la cotidianidad más inmediata, el Dasein fáctico en el cómo de su 'serlo' fáctico ${ }^{3}$.

Sin duda alguna, la fenomenología-hermenéutica encauza la lectura hacia una formación del "sujeto" a partir de la articulación, la fundación y la transformación del mundo en donde actúa y que, a su vez, lo afecta. Después de todo, el Dasein se constituye en relación a un entretejido de vivencias que marcan su existencia. Ciertamente, así se delimitan una serie de condiciones que hacen efectiva toda comprensión del ser y de sí mismo. Acorde con esto el filósofo sostiene en su curso de 1929/30 Die Grundbegriffe der Metaphysik. Welt-Endlichkeit-Einsamkeit:

El hombre no sólo se considera un fragmento del mundo que sucede dentro de él y que conjuntamente lo constituye, sino que el hombre está frente al mundo. Este estar en frente es un tener el mundo como algo en lo que el hombre se mueve, con lo que se confronta, que él domina y de lo que se sirve y a lo que al mismo tiempo está abandonado ${ }^{4}$.

En este texto observamos que la forma que Heidegger elige para hablar del sujeto como un ser que es "en, para y del mundo" concede la posibilidad de realizar una mirada topológica. Puesto que -tal como venimos subrayando- el Dasein es un ser fáctico, cuya esencia se determina por la serie de actividades que emprende en relación a lo dado. $Y$ estas actividades se perfilan, indefectiblemente, de acuerdo a las condiciones espaciales dispuestas en la organización de su entorno ${ }^{5}$. Asimismo, no debe olvidarse que aquel

3 HeIDEGGER, M. Prolegomena zur Geschichte des Zeitbegriffs, GA 20. Frankfurt am Main: Vittorio Klostermann, 1979, pp. 207-208. [Trad. Prolegómenos para una historia del concepto de tiempo, Madrid: Alianza, 2006, p. 194.]

4 HeIDEgGER, M. Die Grundbegriffe der Metaphysik. Welt-Endlinchkeit-Einsamkeit, GA 29/30. Frankfurt am Main: Vittorio Klostermann, 1992, p. 262. [Trad. Los conceptos fundamentales de la metafísica. Mundo, finitud, soledad, Madrid: Alianza, 2007, p. 226.]

5. Esta forma de comprender el mundo está totalmente en consonancia con la lectura husserliana del segundo libro de Ideen zu einer reinen Phänomenologie und phänomenologischen Philosophie 
fenómeno esencial de la temporalidad que fundamenta la estructura del Dasein -la temporalización (Zeitigung)- está asociado directamente con un proceso de espacialización (Räumung) que inscribe la ejecución de dicha estructura en un "lugar propio" (del ser-ahí) y en el marco de un horizonte que prefigura a este ente y su accionar.

Con todo, nos animamos a postular que ese codiciado trasfondo indeterminado y transobjetivo que fija -en el sentido normativo- la experiencia y la comprensión del ser, sólo puede imaginarse en función al aspecto contrastable de lo dado del que se deduce esa zona virtual y potencial de sentido. Con esto queremos decir, que de acuerdo al sondeo fenomenológico no es el trasfondo el que determina a su objeto sino que a partir del objeto vivido (en su sentido práctico) se despliega el haz de posibilidades y remisiones realizables. En efecto, entendemos que lo dado puede obligar al Dasein a reconfigurarse para un acceso apropiado al sentido que asoma. Esta es la consecuencia directa de fundamentar la estructura trascendental en lo fáctico.

Ahora bien, es en este aspecto específico de la teoría heideggeriana donde encontramos la brecha para plantear que la espacialidad (existencial) del Dasein -o sea, el entramado de sentido que se teje según el campo donde acciona- está condicionado por el trato que tiene con lo dado. De forma tal, que la faceta concreta y circunstancial de eso dado no puede omitir los aspectos objetivos de su presentación. Pues bien, no sólo debemos tener en mente el aspecto subjetivo de su organización. De hecho, el método fenomenológico parte siempre por un estudio de lo óntico para un acceso a lo ontológico; claro está esto en la máxima "ia las cosas mismas!" que Heidegger retoma. Por lo que sostenemos que la correlación óntico-ontológica desarrollada en su obra capital, mantiene una dependencia que no remite a condiciones estrictamente formales sino también materiales ${ }^{6}$.

(1952) y la concepción merleaupontyana de Phénoménologie de la perception (1945). Ambas acentúan la importancia de la espacialidad -además de la corporalidad- en la configuración del sentido de la experiencia sensible. Heidegger, para entonces, tuvo acceso a los manuscritos de Husserl que -de cierta forma- concluye en 1928. Por esto, creemos que algo de lo trabajado por Heidegger está en diálogo implícito con estos materiales.

66 Al respecto dice Ramón Rodríguez en Fenómeno e interpretación: "La materia, aquel ingrediente del acto que es responsable de la concreta dirección intencional hacia un objeto en su específica forma de aparecer [...] hace que el acto sea una efectiva presentación de un objeto que aparece en él". (Fenómeno e interpretación. Ensayos de fenomenología hermenéutica, Madrid: Tecnos, 2015, p. 38.) En relación a esto, también es importante reforzar el sentido que tendría una dependencia de lo material. Esto significa, siguiendo a Malpas, que en Sein und Zeit no habría simplemente una dependencia jerárquica del tiempo sobre el espacio sino una dependencia horizontal de lo espacial y lo temporal en la constitución de lo dado. (Véase MALPAS, J. Heidegger's Topology, op. cit., p. 117 ss.) 
A causa de esto se sigue, que lo dado y la situación fáctica tengan una función determinante y fundacional en la constitución de la estructura de nivel trascendental. Ambas se hallan en correspondencia con la noción de lugar, puesto que toda dación concreta se materializa en una localización (Ortschaft) que dispone de cierta manera al objeto en escena. Tal como lo entiende Jeff Malpas en su escrito Heidegger's Topology. Being, Place, World: el ser es un efecto del lugar ${ }^{7}$, es decir, de un emplazamiento emerge lo dado ${ }^{8}$. En tal caso, a pesar de que Heidegger prefiera al tiempo porque capta mejor la dinámica y lógica de la manifestación del ser, insistimos en que es el espacio el que logra reunir y en-caminar (Be-wëgen) a su puesto aquello que se muestra -aunque sea bajo la opacidad de una forma diferenciada (entitativa). De esto resulta, que la temporalidad originaria que consolida la propuesta del filósofo en Sein und Zeit (1927) y dirige la investigación hacia la cuestión del ser, implica cierta espacialidad que también condiciona al Dasein y deja su sello en los caracteres óntico-ontológicos. Esto lo explicaremos una vez que penetremos en el análisis de la espacialidad del Dasein que en el período de Marburgo Heidegger plasma.

En síntesis, básicamente pretendemos divisar por qué habría una relación entre lo espacial y el sentido objetivo de lo dado. Pues bien, el proceso de espacialización contemplaría estos aspectos materiales del orden sensible que co-determinan la estructura del Dasein. Ahora bien, lo problemático de este asunto es fundamentar que este tipo de vínculo entre lo espacial y lo material tiene, además de un sentido derivado -como lo ve Heidegger- un sentido originario que la fenomenología debe explicitar. El sentido existencial, que Heidegger desarrolla en este período de Marburgo, podría decantar en una interpretación originaria de lo espacial. No obstante, hay que ver si el filósofo logra hacerlo.

\section{Revisión del proceso de espacialización y su sentido en la analítica existencial}

Heidegger anticipa el sentido existencial de la espacialidad en el curso Prolegomena zur Geschichte des Zeitbegriffs mediante un des-alejamiento (Ent-fernung), zona (Gegend) y orientación (Orientation) del Dasein en el mundo. De esta forma describe la movilidad de este ente, esbozando el campo en el que se cristaliza la constitución de su estructura. Particularmente, la mundaneidad del mundo, su comparecencia (Begegnis) y el trato (Umgang) con el entorno visibilizan el espacio y su posición en él. En definitiva, y tal como

7. Dastur señala sobre este asunto que el espacio debe entenderse como donación, una puesta en el espacio (Einräumen). (Cfr. Heidegger y la cuestión del tiempo, Buenos Aires: Del Signo, 2006, p. 54) 8. MALPAS, Heidegger's Topology, op.cit. pp. 13-14. 
dijimos, Heidegger indaga acerca del proceso de espacialización del Dasein a través de su conducta en relación a lo dado. Pues bien, todo des-alejamiento, zona y orientación en el mundo se asumen respecto de algo.

Bajo esta perspectiva, el objeto al cual el Dasein se dirige no se computa en el espacio a partir de una serie de coordenadas de localización geométrica. Por consiguiente, el tipo de relación que entabla con lo dado no se rige por los parámetros cuantificables de un proceso matemático. Ya lo indica al inicio del $\S 19$ de los Prolegomena... en el cual especifica que el sentido al que se refiere cuando habla del ser-en o el estar-siendo-en (In-Sein) del Dasein no tiene que ver con un estar dentro-de un espacio -como se dice de los objetos físicos-corpóreos ${ }^{9}$. Por el contrario, y tal como se consolida en Sein und Zeit, el ser-en del Dasein es vivir, habitar y un estar familiarizado-con ${ }^{10}$. En tal caso, “...se da un sentido local auténtico, que, no obstante, sigue siendo esencialmente diferente del estar espacial de una cosa en otra"11.

Si bien el sentido local del ahí por el que Heidegger alude implícitamente a la espacialidad termina interpretándose por medio de la categoría de la temporalidad porque ese ahí es ocasional (jeweilig). Aún así, al filósofo le sigue preocupando establecer algún estilo de

9. Jeff Malpas señala que el concepto de lugar (Place-Ort) puede leerse ónticamente en un sentido derivado, si se lo comprende como un punto o posición en el espacio físico extendido. No obstante, Heidegger apunta con él a una dimensionalidad originaria y abarcativa que, en el segundo período de su pensar, conlleva la reunión y la relacionalidad de lo que se emplaza. Falta descubrir qué sentido tiene el concepto de lugar en su obra capital y los escritos en torno a ella. Más que nada, este concepto debe abordarse con la intención de rescatar el aspecto situacional que Heidegger desarrolla a partir de los términos: Stelle, Platz y Stätte. Es decir, por fuera del sentido de un lugar en un espacio extendido.

10. Dastur rescata que cuando Heidegger define el modo de estar del Dasein como In-Sein, el "In" alemán procede de "innan" que quiere decir habitar. Aquí no se habla de un estar-en (que contiene) sino de un sentido existencial de la proximidad, el contacto y el encuentro posible con los entes. Dastur afirma que la espacialidad no debe eludirse porque el Dasein no es un espíritu que mantendría relaciones extrínsecas con el espacio. Aunque, entiende que tal espacialidad tampoco debe remitirse al cuerpo porque si no se pierde su sentido existencial y ontológico. (Véase DASTUR, Heidegger y la cuestión del tiempo, op. cit., p. 54) En tal caso, según Dastur, el cuerpo sólo puede interpretarse en el pensamiento de Heidegger en sentido fisicalista. Pues bien, el filósofo no menciona el sentido vivido del cuerpo; aquél que Merleau-Ponty afianza y que el propio Husserl en Ideen II ya presenta en detalle. Consideramos que por medio del cuerpo vivido se abre, efectivamente, el acceso y comprensión de esta otra espacialidad originaria de los actos. Lamentablemente, Heidegger no quiso tomar esta vía interpretativa en el período de Marburgo.

11. HeIDEgGer, M. Prolegomena, op. cit., p. 213 [Trad. Prolegomenos, op. cit., p. 199] De hecho, el concepto de lugar (topos) que Heidegger retoma de la Física de Aristóteles representa la idea de que cada ente, de acuerdo a su condición natural, posee un lugar en el cosmos y se distingue de otros entes por los límites que lo diferencian (podría decirse, los contornos que envuelven a la figura -dado que el estagirita no concebía la idea de un vacío en el que se dispongan los entes). 
medida para la dimensión de lo ocasional. De hecho, se pregunta: “¿Cuál es la amplitud"2 ocasional de lo descubrible correspondiente a un determinado modo de ser-en del Dasein en su mundo?"13. O sea tiene una preocupación por registrar la dimensión de lo espacial. Entonces, entiende que esta dimensión que comprende formalmente el estar-siendo-enel-mundo y que concibe como la mundaneidad reviste el carácter de una espacialidad diferente, es más, de una localidad (Örtlichkeit) que, literalmente, "desempeña un papel especialmente notable"14 para la constitución del mundo. A esta mundaneidad le quiere devolver el lugar y espacio auténticos que por derecho le corresponde y que tiene dentro de la estructura del Dasein ${ }^{15}$. Es más, Heidegger sostiene en el apartado de la espacialidad lo siguiente:

Sólo cuando se ha visto la mundaneidad del espacio y la espacialidad primaria, es decir, cuando se ha entendido el espacio del mundo-en-torno y su trama estructural junto con el Dasein, está uno en situación de evitar una vía que (...) caracteriza el espíritu y el ser de lo espiritual (...) de manera negativa, por oposición al espacio ${ }^{16}$.

Heidegger señala, llamativamente, que el Dasein es espacial y que no existe razón alguna para oponerse a ello, al menos si nos abocamos a dilucidar su sentido originario.

Por lo tanto, Heidegger plantea en los Prolegomena... que el hecho de verse en la necesidad de excluir un sentido determinado de espacialidad es una señal clara de que en estos fenómenos interviene otro sentido de la misma. Por esto, se ocupa de la tarea de mostrar otra manera de dar con lo dado para develar cómo procede y se constituye el mundo del Dasein, es decir, cómo se espacializa (räumt). Pues bien, la espacialidad es un elemento constituyente del mundo -Heidegger así lo afirma en el §25 del curso. Por este motivo, es que tiene que introducirse en el trato con lo dado en la cotidianidad para develar la estructura del proceso de espacialización. Así pues, el filósofo aclara en el curso que:

12. Sobre esta cuestión afirmamos que si Heidegger quiere captar la amplitud (Gröbe) que tiene la dimensión en donde se emplaza el Dasein en su ahí, entonces le interesa indagar -aunque sea de manera originaria- en el carácter extensivo de la misma. Ya sabemos que en los escritos posteriores a la Kehre (tales como Das Wesen der Sprache-GA 12) Heidegger dice que la diferencia (como contraposición de lo uno y lo otro) es la que mide o mesura (Er-messen) la medida de su esencia.

13. HeIDEgGer, M. Prolegomena, op. cit., p. 218 [Trad. Prolegomenos, op. cit., p. 204]

14. HEIDEGGER, M. Ibidem, p. 226 [Trad. Ibidem, p. 211]

15. Cfr. HEIDEGGER, M. Ibidem, p. 230 [Trad. Ibidem, p. 214]

16. HEIDEGGER, M. Ibidem, p. 307 [Trad. Ibidem, p. 280] 
...lo que tenemos es el ente dado en su inmediatez, la cosa del mundo, y el modo de acceder a ella es la percepción sensible [...] la cosa tal como se presenta de entrada en el mundo [es] una cosa de la naturaleza, cuyo estrato básico es la materialidad, pero a la vez provista de predicados de valor ${ }^{17}$.

Sin embargo, más adelante plantea que este tipo de mirada práctica sobre la cosa está teñida por las facultades que una antropología o biología le acredita al hombre. De esta manera, la percepción hace a la cosa percibida ${ }^{18}$.

En esta línea de interpretación, la manifestabilidad de lo dado está determinada por el tipo de acceso ${ }^{19}$. De este modo, es probable que el verdadero ser de la cosa del mundo entorno (Umweltding) se pase por alto. Este hilo argumentativo deja en evidencia la necesidad de detenerse en lo dado para poder describirlo sin desfigurar su sentido. Con esto, entendemos que el recorrido vira -o intenta hacerlo- de la primacía de las condiciones de posibilidad de la experiencia de base subjetiva hacia a una serie de condiciones del objeto (no objetivado). Estas condiciones propias del objeto -que no se reducen a una teoría del conocimiento- presentan lo que en Sein und Zeit y en este curso previo se concibe como significatividad (Bedeutsamkeit). De hecho, el sentido de lo dado en su forma originaria se despliega para el Dasein de acuerdo a cómo vivencia -de manera cotidiana e inmediata bajo su utilidad, practicidad o para-qué y en el marco remisional de una serie de relaciones absortas- tal significatividad. Con esto se va arribando a la tesis de que la espacialidad originaria de la práctica del Dasein, se realiza a partir del sentido pre-objetivo de lo dado. Un sentido que se aparta de la lectura teórica objetivante pero que pierde de vista, a nuestro juicio, el sentido objetivo al quedarse exclusivamente en la recepción y formas de interpretación de eso dado. Por ende, consideramos que hay algo en "la cosa del mundo" que excede al condicionamiento del tipo de acceso y debe descubrirse sin quebrantar los matices de su procedencia.

17. HEIDEGGER, M. Ibidem, p. 246 [Trad. Ibidem, p. 228]

18. Rodríguez aclara que Heidegger en el período de Marburgo, especialmente en los textos en torno a Sein und Zeit, se preocupa por el sentido perceptivo y explica que éste "...no se refiere a los rasgos meramente ónticos del objeto, a sus predicados reales, sino a lo que aparece en la percepción justamente en tanto que aparece así en ella" (RODRÍGUEZ, R. Fenómeno e interpretación, op. cit., p. 38)

19. Sabemos que en el período de Marburgo Heidegger analiza el proceso perceptivo y el sentido de lo que se manifiesta de acuerdo a como lo recibe el Dasein e, incluso, a través de la accesibilidad que la estructura subjetiva tiene. No obstante, para Rodríguez el propio concepto de manifestabilidad es complejo y un poco ambiguo porque "...no sabemos si es una propiedad de otro orden de la cosa, si es algo ajeno a ella o si es un rendimiento de la conciencia". (Ibidem, p. 42) 
Ahora bien, este vínculo con lo dado, más allá de la manera puntual de manipular un útil, se vislumbra en los Prolegomena... como "resistencia" (Wiederstand). Heidegger sostiene que su postura se asemeja al tratamiento que Scheler hace respecto de lo dado, puesto que se vive de modo originario en el acto del desear, el instinto y la voluntad simplemente como una resistencia o contraimpulso. Esta resistencia debería aludir, para nosotros, a las notas del objeto que el sujeto experimenta en un estado previo al acto deliberativo o intencional. Esto comportaría una suerte de recepción pasiva del sentido originario que asoma en el propio objeto y en su forma de presentarse. No obstante, para Heidegger esa resistencia que nos ofrece lo dado se subordina al estrato formal del cuidado y la significatividad porque, según él, de la resistencia de dos entes no surge el mundo ${ }^{20}$. Así que a pesar de que el fenómeno de la resistencia tiene en cuenta a lo dado bajo el carácter de su materialidad primitiva, Heidegger argumenta que una resistencia se ofrece para la actividad de un Dasein.

En resumen, la idea de Heidegger se acota a mostrar el sentido de lo dado por fuera de toda facultad subjetiva objetivante, por ello el filósofo se concentra excluisivamente en la descripción de la mundaneidad y su sentido remisional. En tal caso, la constitución de la mundaneidad se produce a partir del des-alejamiento, la zona y la orientación, que mencionamos en un principio. Éstos conllevan una cercanía (Nähe) y direccionalidad (Ausrichtung) por parte del Dasein que no se posiciona de forma calculadora. Al respecto, Heidegger habla de una hermenéutica y valoración cotidianas ${ }^{21}$. En el curso no ofrece una explicación muy extensa respecto de estos existenciales, pero sí señala que son versiones originarias que se desmundanizan cuando se introduce la idea de una distancia "extensiva". Por el contrario, las tres variantes del comportamiento espacial -a las que allí dedica algunas páginas- sugieren una forma puntual de disponerse frente a los objetos "alejados". Esta forma de disposición, que veremos se supedita al tiempo, genera un trato con el entorno que reposiciona sus objetos de acuerdo a un objetivo vivencial determinado. El objetivo emerge del sentido inherente al acto, sin producir una reflexión que corte el vínculo inmediato con lo dado. Por ejemplo el filósofo dice:

El camino que recorre uno todos los días y del cual quizás se sabe en términos objetivos cuánto tiempo lleva recorrerlo puede resultar cada día diferente, más o menos largo. Esto no es más que el reflejo de que soy yo el que recorre el camino y lo recorro en cada caso como des-alejamiento mío, en el cual me alejo de algo o me acerco a algo que yo mismo he puesto en

20. HeIDEGGER, M., Prolegomena, op. cit., p. 304 [Trad. Prolegomenos, op. cit., p. 277]

21. HEIDEGGER, M. Ibidem, p. 36 [Trad. Ibidem, p. 288] 
mi ocupación, algo que no es un punto cualquiera del espacio, sino en lo que estoy ya de antemano en mi Dasein ${ }^{22}$.

En este sentido, la medida de las distancias producidas por el des-alejamiento, la zona en la que se mueve el Dasein y la orientación que establece frente a lo dado, no se determina por medio de una actitud objetivante. Asimismo, la reflexión no obstruye la dinámica remisional de lo vivido para pautar algún tipo de modificación o redirección del accionar en el entorno. El Dasein se maneja bajo una comprensión pre-teórica en la cual lo dado se experimenta a través de un sentido que dispone las cosas en función a cómo afectan a la conducta y el propósito, que -al parecer- no fue calculado, diseñado o estipulado más allá del estar-siendo-en-el-mundo en su versión primaria. La ocasionalidad de lo vivido y el carácter singular de la experiencia, que remiten a un Dasein en particular, son las notas definitorias de este estilo de medición de lo espacial.

Ahora bien, ¿̇esta manera de comprender el espacio de forma originaria está fundada en la temporalidad? Es decir, ¿̇se ciñe a cómo experimentamos el tiempo respecto de un movimiento ocasional del Dasein? Heidegger sostienen en estos pasajes del curso que, de acuerdo a como vivenciamos temporalmente un objeto se comprende de cierta forma la duración del des-alejamiento. La diferencia de las duraciones se basa en el ocuparse (el existencial del cuidado-Sorge) y en cómo está uno en el tiempo. Ante esta salida heideggeriana, consideramos que el tratamiento del problema de la espacialidad se deforma porque el filósofo introduce en la interpretación cotidiana del movimiento efectuado por el Dasein -o sea la espacialización que comprende el trato con lo dado- al tiempo. Cuando habla de otro estilo de espacialización que no atiende al cálculo de las distancias se remite a la "duración". Esta es la versión métrica del tiempo que se emplea para establecer cómo se vivencia el des-alejamiento.

En tal caso, ¿por qué el des-alejamiento se experimenta temporalmente como duración? Heidegger afirma, en el análisis de este comportamiento, que el tacto no es el sentido que permite mostrar mejor el alejarse o acercarse a las cosas, y que -en efecto- es el andar el que mejor refleja las distancias. Aquí parece encontrarse, a nuestro juicio, la pérdida del hilo argumentativo porque la espacialidad de tal movimiento se subordina a la temporalidad experimentada por el agente-móvil; ya no prima la experiencia de lo dado y su materialidad. Ahora, la medida o condición la pone el Dasein. No obstante, si la extensión es una propiedad objetivada -en su sentido teórico-, la duración también debe serlo. Entonces, como la duración tiene una versión originaria de expresión en la interpretación del filósofo: ¿por qué no ha de tenerla la extensión? ¿Por qué el joven

22. HEIDEGGER, M. Ibidem, p. 317 [Trad. Ibidem, p. 289] 
Heidegger no apela a la extensión para explicar la distancia que se reduce con el desalejamiento? Heidegger prefiere plantear la medida en términos de "duración" ya que, al parecer, la "extensión" refiere a los objetos o a la medida "objetiva" de la distancia y, en cambio, el tiempo apunta mejor a cómo experimenta el Dasein el des-alejamiento. El tiempo es la versión "subjetiva" de ese trayecto que captamos para él como "duración". Por lo tanto, el espacio se temporaliza.

Volviendo a los sentidos y las preferencias de Heidegger, cuando rechaza el oído, la visión y el tacto para ejemplificar el des-alejamiento, y plantea al andar como el tipo de percepción que mejor atiende a la proximidad de las cosas, encontramos nuevamente esa necesidad de registrar cómo afectan las cosas sin remitirnos a la materialidad de lo dado. Por ende, la lectura inicial de la espacialidad que se definía como un "ser-en" o un "estarsiendo-en-el-mundo" deja de lado sus rasgos topológicos para focalizarse en los aspectos modales que indican cómo se siente el Dasein y cómo vive temporalmente tal movilidad. Esto genera una recaída en una lectura subjetiva del movimiento que desfigura el proceso de espacialización, al desconsiderar el aporte de lo dado en la conformación del sentido de esta práctica. De esta manera, el comportamiento del Dasein que se avistaba con el desalejamiento, la zona y la orientación, ya no vislumbra esa dimensión originaria en donde se emplazaba el Dasein (el ahí de su ser) en relación a lo dado. Desgraciadamente, el asunto de la espacialidad que también hacía su aporte en la constitución de la estructura del Dasein queda olvidado.

Más adelante, Heidegger profundiza (aunque, a su vez, restringe más) el sentido de este tipo de movilidad (Bewegtheit) característica del Dasein en el curso de 1927, Die Grundprobleme der Phänomenologie, por medio de los conceptos de distensión (Dehnung), continuidad (Stetigkeit) y dimensión (Dimension) -que acompañan su interpretación de la Física de Aristóteles; especialmente en $\S 19$ (a. $\beta$ ). En esta oportunidad también prevalece el sentido temporal del movimiento del Dasein, que de cierta manera exhibe otra forma de entender el proceso de espacialización. Esto sucede ya que refuerza el carácter vital y afectivo de este tipo de movimiento, por sobre la presunta consideración fisicalista que tendría la versión extensiva de lo espacial -al entenderlo como el tránsito de un lugar a otro.

En este curso Heidegger opina que si se piensa al tiempo de modo instrumental, como medida a través de lo numerado, se lo espacializa. (Nos preguntamos: ¿por qué cuando pensó como duración al movimiento no resultó éste deformado por el tiempo?) Esta lectura degrada de entrada el sentido del espacio que se estaba gestando. Ahora bien, ¿̇por qué Heidegger intuye la posibilidad de pensar al tiempo de manera originaria y, contrariamente, no acaba de pensar lo mismo acerca del espacio? Quizás en este período 
de su pensar, no hay posibilidad de admitir una lectura que exceda la posición moderna. Y, sin embargo, la analítica existencial tiene una fuerte base topológica, en el sentido aquí rescatado.

Entonces, aquel matiz existencial que se abría con la revisión ontológica de la espacialidad de la estructura del Dasein y su funcionamiento, se desvirtúa para dar paso a un seguimiento meramente formal de la manifestación del ser, que considera a lo material/óntico como un traspié al que el método se ve forzado. El tipo de prolongación o distanciamiento (Erstreckung) y transformación de la estructura del Dasein que describe como distensiva -en vez de extensiva- domina la exposición y termina por confinar a un plano derivado la espacialidad. Esto acontece porque cuando plantea al Dasein en movimiento lo interpreta por fuera del espacio y en función de una lectura de la traslación o el desplazamiento cualitativa (esencial). Heidegger sostiene que con el tiempo, el movimiento genera un tránsito de un lugar a otro pero no en el orden de lo espacial ${ }^{23}$. De este modo, se opone a introducir la categoría de lo espacial a la hora de explicar la relación entre el tiempo y el movimiento.

Aunque, la imposibilidad de una omisión del pasaje metodológico por lo "derivado", lleva a que en los conceptos de continuidad y dimensión que fundamentan lo temporal, la espacialidad se asome (o se entrometa como dice Malpas). Esto ocurre cuando Heidegger retoma de Aristóteles el aspecto de un lugar (topos) que abarca el movimiento sin ser él mismo movimiento, es decir, sin verse afectado por la secuencia calculable de la trayectoria. Dado que la idea del lugar propio y natural del móvil -que procede de Aristóteles- desliza nuevamente el sentido originario hacia lo material, ya que la disposición "temporal" del movimiento del Dasein se sostendría gracias a un horizonte singular y fáctico que la habilita (el mundo ${ }^{24}$, y este horizonte de avistamiento es espacial.

En otras palabras, cuando el ente se "encuentra" en el ámbito propicio de manifestación y está "dis-puesto" de forma apropiada estarían dadas las condiciones óptimas para la captación. No pueden negarse que estas condiciones están vinculadas directamente con una manera espacial de vivenciar lo dado. No obstante, el concepto de horizonte nuevamente se temporaliza y, por ende, se enmascara aquel "sitio" (Platz) bajo la idea de un "instante" (Augenblick), que ciertamente establece la proximidad a la donación. De este modo, Heidegger subordina la espacialidad a la condición temporal del Dasein porque -como ya advierte al final del apartado 25 de los Prolegomena- todo des-alejamiento, bajo

23. HeIDEgGeR, M. Die Grundprobleme der Phänomenologie, GA 24, Frankfurt am Main: Vittorio Klostermann, 1989, p. 343. [Trad. Los problemas fundamentales de la fenomenología 1927, Madrid: Trotta, 2000, p. 294]

24. Véase HeIDEgGeR, M. Grundprobleme, op. cit., p. 360 [Trad. Problemas, op., cit., p. 307] 
la forma de la lejanía o la cercanía, se dispone en una temporalidad ocasional y singular.

De allí se sigue, que el existencial de la zona se presente en Sein und Zeit como un entramado de relaciones que establecen la pertinencia (Hingehörigkeit) de acuerdo a la ocupación del Dasein. Pues bien, para que el Dasein pueda efectuar movimiento alguno en dirección a un objeto, éste debe presentarse de antemano en su significatividad. EI hecho de estar-siendo siempre ya en-el-mundo condiciona de manera previa nuestra existencia. En este sentido es que la materialidad se pierde frente a la determinación ontológica que genera el horizonte indefinido o transobjetivo que sostendría a lo dado. La estructura pre-ontológica del Dasein y su manera de anticiparse constituyen un tipo de orientación que no es meramente espacial, es decir que no tiene que ver con cómo organizamos la zona bajo sus condiciones materiales. Este ordenamiento procedería de la capacidad de adelantarse a lo dado en un sentido teleológico.

\section{Conclusión: La subordinación del espacio a una interpretación temporal}

Finalmente, debemos admitir que en el caso del período de Marburgo el movimiento que efectúa el Dasein se define a partir de la trascendencia y la proyección de este ente hacia el plano ontológico ${ }^{25}$. De esto se sigue, que la interpretación de la espacialidad que constituye el modo de estar-siendo-en-el-mundo dependa de la temporalidad ${ }^{26}$, pues la caracterización ex-tática de este movimiento está directamente relacionada con la anticipación compresora que se obtiene de la unidad de la estructura a partir del cuidado y el tiempo. Si bien esta impresión es la que prevalece, subrayaremos que el movimiento "vital y afectivo" en algún punto y, para ser precisos en un comienzo, debe registrarse en el orden de lo óntico. Por consiguiente, requiere visualizarse en la experiencia sensible a través de aquello que se mueve (el Dasein), o sea, en un emplazamiento preciso. Así pues,

25. Más adelante, con la transformación de su pensar, estos conceptos espaciales reaparecen bajo el contexto más amplio de un ámbito o región (traducción preferida aquí para Gegend) que además de un desplazamiento del Dasein involucra, en el mismo nivel, un emplazamiento del Seyn -específicamente en el ensamble de la fundación (Gründung) punto (d) "El espacio-tiempo como abismo" del texto Beiträge zur Philosophie. Vom Ereignis. Por lo tanto, desplazamiento y emplazamiento son dos conceptos enlazados (en la Zeit-Räum Gefüge). De acuerdo a la interpretación de Malpas respecto a la función de la dependencia entre espacio y tiempo -entre otras- (nota siguiente), quizás se puede decir que en este segundo período de Heidegger la dependencia sea mutua.

26. Jeff Malpas realiza un análisis profundo acerca de los sentidos que en la obra de Heidegger debería tener tal dependencia y fundamentación. Allí habla de una dependencia mutua o jerárquica entre distintos existenciales que Heidegger no deja bien clara. (Véase, Heidegger's Topology, op. cit., pp. 114-118) 
la idea heideggeriana de una movilidad temporal se alinea de entrada con un espacio que permite constatarla.

No obstante, en el $\S 70$ de Sein und Zeit Heidegger afirma que el hecho de que se dé la estructura en un tiempo y espacio no es lo que comprende como su sentido existencial ${ }^{27}$. Heidegger sostiene que el Dasein jamás está en el espacio óntico, sino que es en el espacio gracias a la mundaneidad que lo determina y de acuerdo al ordenamiento significativo de su ocuparse. Vimos que tal ordenamiento de la zona se configura por la orientación temporal del Dasein. En este caso, el espacio toma posesión extáticamente. Tan sólo en base a la temporalidad extático-horizontal es posible la irrupción del Dasein en el espacio ${ }^{28}$.

A pesar de esta fuerte afirmación, Heidegger no podrá borrar del mapeo ontológico a la espacialidad. Pues, si bien la comprensión que tiene del espacio en su versión existencial emerge de la temporalidad, no puede obviar el hecho de que toda la "analítica existencial" produce una descripción del modo de ser de un Dasein situado y orientado dentro de un entramado de sentido que emerge y se realiza en la facticidad misma. Por lo tanto, si pretende develar cómo se produce el sentido en la experiencia y se asienta en una forma concreta no puede eludir aquellos aspectos ónticos que la delimitan y que adquieren cierta configuración de acuerdo a factores que exceden la pre-disposición formal del Dasein. Heidegger dice:

...no sólo se comprende [el Dasein] a partir de los entes a la mano que son parte de su ocupación [...] sino que toma de las relaciones espaciales, los hilos conductores para la articulación de lo comprendido ${ }^{29}$.

\section{Bibliografía}

ARISTÓTELES: Física, Madrid: Gredos, 1995.

BLATTNER, W.: Heidegger's temporal idelism, New York: Cambridge University Press, 1999. CROWELL, S., MALPAS, J. (eds.): Transcendental Heidegger, California: Stanford University Press, 2007.

DASTUR, F.: Heidegger y la cuestión del tiempo, Buenos Aires: Del Signo, 2006.

27. HeIDEgGeR, M. Sein und Zeit, Tübingen: Max Niemeyer Verlag, 1960, p. 367. [Trad. Ser y tiempo, Chile: Universitaria, 1997, p. 383]

28. HEIDEGGER, M. Ibidem, p. 369 [Trad. Ibidem, p. 384] El subrayado es de Heidegger.

29. HEIDEGGER, M., Ibidem., p. 369 [Trad. Ibidem, p. 385] 
DREYFUS, H.: Ser-en-el-mundo. Comentario a la división I de Ser y Tiempo de Martín Heidegger, Santiago de Chile: Cuatro Vientos Editorial, 1996.

HEIDEGGER, M.: Unterwegs zur Sprache, GA 12, Frankfurt am Main: Vittorio Klostermann, 1985. [Trad. De camino al habla, trad. Yves Zimmermann, Barcelona: Serbal, 1987.]

HEIDEGGER, M.: Prolegomena zur Geschichte des Zeitbegriffs, GA 20, Frankfurt am Main: Vittorio Klostermann, 1979. [Trad. Prolegómenos para una historia del concepto de tiempo, Madrid: Alianza, 2006.]

HEIDEGGER, M.: Logik. Die Frage nach der Wahrheit, GA 21, Frankfurt am Main: Vittorio Klostermann, 1976. [Trad. Lógica. La pregunta por la verdad, Madrid: Alianza, 2004.]

HEIDEgGER, M.: Die Grundprobleme der Phänomenologie, GA 24, Frankfurt am Main: Vittorio Klostermann, 1989. [Trad. Los problemas fundamentales de la fenomenología 1927, Madrid: Trotta, 2000.]

HEIDEGGER, M.: Die Grundbegriffe der Metaphysik. Welt-Endlinchkeit-Einsamkeit, GA 29/30, Frankfurt am Main: Vittorio Klostermann, 1992. [Trad. Los conceptos fundamentales de la metafísica. Mundo, finitud, soledad, Madrid: Alianza, 2007.]

HEIDEgGeR, M.: Beiträge zur Philosophie (Vom Ereignis), GA 65, Frankfurt am Main: Vittorio Klostermann, 1994. [Trad. Aportes a la filosofía. Acerca del evento, trad. Dina Picotti, Buenos Aires: Almagesto-Biblos, 2003.)

HEIDEGGER, M.: Sein und Zeit, Tübingen: Max Niemeyer Verlag, 1960. [Trad. Ser y tiempo, Chile: Universitaria, 1997]

HUSSERL, E.: Ideen zu einen reinen Phänomenologie und phänomenologischen Philosophie. Zweites Buch: Phänomenologische Untersuchungen zur Konstitution, The Hague: Martinus Nijhoff, 1952.

MALPAS, J.: Heidegger's Topology. Being, Place, World, Cambridge: The MIT Press, 2006.

MERLEAU-PONTY, M.: Phénoménologie de la perception, Paris: Gallimard, 1945.

ROCHA DE LA TORRE, A.: "El concepto de cercanía en Martin Heidegger", Eidos, N 7, 2007, pp. 48-86.

RodrígueZ, R.: Fenómeno e interpretación. Ensayos de fenomenología hermenéutica, Madrid: Tecnos, 2015.

QUENAYA MENDOZA, C.: "De Aristóteles a Heidegger un recorrido del lugar a la espacialidad del Dasein", Phainomenon, Vol. 11, N 1, 2012, pp. 63-69. 
VIGo, A.: Arqueología y aleteiología, Buenos Aires: Biblos, 2008.

VOLPI, F.: Heidegger y Aristóteles, Buenos Aires: FCE, 2012. 